\title{
Jordan Canonical Forms of Riordan Arrays
}

\author{
Roksana Słowik(i)
}

\begin{abstract}
We consider the possible Jordan canonical forms of Riordan arrays. We prove that there are, in fact, only two such forms. Moreover, the transition matrix is in the Riordan group only in the case when the given Riordan array has one of some three specific forms.
\end{abstract}

Mathematics Subject Classification. 15A21, 15B99, 39B22.

Keywords. Riordan array, Jordan canonical form, Jordan base.

\section{Introduction}

Riordan arrays [7] are infinite lower triangular matrices $R=\left[r_{n k}\right]_{n, k \in \mathbb{N}_{0}}$ that are identified with pairs of formal power series $(d(t), h(t))$ as follows:

$$
r_{n k}=\left[t^{n}\right] d(t)(h(t))^{k} \quad \text { for all } n, k \in \mathbb{N}_{0},
$$

where $d \in \mathcal{F}_{0}=\left\{d_{0}+d_{1} t+d_{2} t^{2}+\cdots: d_{0} \neq 0\right\}, h \in \mathcal{F}_{1}=$ $\left\{h_{1} t+h_{2} t^{2}+\cdots: h_{1} \neq 0\right\}$, and $\left[t^{n}\right] f(t)$ denotes the $n$th term of the series $f(t)$. condition (1) means that the series $d(t)(h(t))^{n}$ is the generating function of the $n$th column. These pairs are multiplied according to the rule

$$
\left(d_{1}(t), h_{1}(t)\right) *\left(d_{2}(t), h_{2}(t)\right)=\left(d_{1}(t) \cdot d_{2}\left(h_{1}(t)\right), h_{2}\left(h_{1}(t)\right)\right) .
$$

The set of all Riordan arrays with the multiplication $*$ forms a group called the Riordan group which will be denoted by $\mathcal{R}$.

In [6] the authors show how to compute some functions of truncated Riordan arrays using their Jordan canonical form. For the sake of clarity, let us present the definition: the triangular matrix of the form $J_{R}=X^{-1} R X$, with $X$ being a Riordan array, that is a direct sum of Jordan cells (possibly also infinite) is called the Jordan form of Riordan array $R$. If we allowed $X$ be any $\mathbb{N}_{0} \times \mathbb{N}_{0}$ matrix with a finite number of nonzero entries in each row, then the generalized Jordan form would for sure exist [5]. However, would they 
also be Riordan arrays? And in which cases their transition matrices would be Riordan arrays as well? In this paper we answer these two questions. Namely, we will prove that the following two hold.

Theorem 1. The only Jordan forms that are Riordan arrays are

1. diagonal matrices $(\alpha, \beta t)=\left[\alpha \cdot \beta^{n} \cdot \delta_{n, k}\right]_{n, k}(\alpha, \beta \in \mathbb{C})$,

2. Jordan cells $(\alpha+t, t)(\alpha \in \mathbb{C})$.

Theorem 2. Riordan array $R=(d(t), h(t))$ with

$$
d(t)=\alpha+d_{1} t+d_{2} t^{2}+\cdots, \quad h(t)=\beta t+h_{2} t^{2}+h_{3} t^{3}+\cdots,
$$

has transition matrix $X$ which is a Riordan array if and only if one of the following holds

1. $R=\alpha \tilde{R}$ where $\tilde{R}$ is an array of finite order in the Riordan group,

2. $\beta$ is not a root of unity,

3. $R=(d(t), t)$ with $d_{1} \neq 0$.

Moreover, in the first two cases of the above theorem the Jordan form of $R$ is a diagonal matrix, whereas in the third case $J_{R}=(\alpha+t, t)$, i.e. $J_{R}$ is a single Jordan cell.

\section{Proofs}

The proof of Theorem 1 is not very complicated. To derive it, let's first draw the attention to the main diagonal of an arbitrary Riordan array. If $R=(d(t), h(t))$ is such that $d(t)=\alpha+d_{1} t+d_{2} t^{2}+\cdots, h(t)=\beta t+h_{2} t^{2}+h_{3} t^{3}+\cdots$, then we can distinuish the following possibilities.

Remark 1. Let $R$ be a Riordan array. Then the entries from the main diagonal of $R$ are either

1. constantly equal to some $\alpha$ or

2. they form a periodic sequence, i.e. $\alpha, \alpha \beta, \alpha \beta^{2}, \ldots, \alpha \beta^{n-1}, \alpha, \alpha \beta, \ldots$ or

3. they form a sequence of the form $\alpha \cdot \beta^{n}$ with $\beta^{n} \neq \beta^{m}$ for $n \neq m$.

It can be noticed that the 'form' of the main diagonal depends (mostly) on the coefficient $\beta$.

Now we can prove the first result.

Proof of Theorem 1. Let's consider an arbitrary $R=\left[r_{n k}\right]=(d(t), h(t))$. By Remark 1 we have three possibilities.

1. If $r_{n n}=\alpha$ for all $n \in \mathbb{N}_{0}$, then the entries of the Jordan form of $R$, denote it $J_{R}=\left[j_{n k}\right]$, can be expressed as

$$
j_{n k}= \begin{cases}\alpha & \text { if } k=n \\ 1 & \text { if } k=n-1 \text { and } n \in A \\ 0 & \text { if } k=n-1 \text { and } n \notin A \\ 0 & \text { otherwise }\end{cases}
$$


for some set $A \subseteq \mathbb{N}$. The above form implies that we should have

$$
d(t)(h(t))^{n}=\left\{\begin{array}{l}
\alpha t^{n}+t^{n+1} \quad \text { for } n \in A \\
\alpha t^{n} \text { for } n \notin A .
\end{array}\right.
$$

If $A$ contains at least two consecutive numbers, Eq. (2) yields $h(t)=$ $t, d(t)=\alpha+t$. Consequently $J_{R}$ is a Jordan cell and $A=\mathbb{N}$. If the complement of $A-A^{\prime}$ contains at least two consecutive numbers, Eq. (2) forces $h(t)=t, d(t)=\alpha$, and in this case $J_{R}$ is a scalar matrix (i.e. of the form $\alpha I$ ), whereas $A=\emptyset$. Suppose that neither $A$ nor $A^{\prime}$ contain two consecutive numbers, from the equalities

$d(t)(h(t))^{n}=\alpha t^{n}+t^{n+1}, \quad d(t)(h(t))^{n+1}=\alpha t^{n+1}, \quad d(t)(h(t))^{n+2}=\alpha t^{n+2}+t^{n+3}$, one gets $h(t)=\frac{\alpha t}{\alpha t+1}=\alpha t+t^{2}$, i.e. a contradiction.

2. If the main diagonal of a Riordan array is periodic, then the main diagonal of any Riordan array that is similar to it (even if the similarity matrix is not Riordan) is also periodic. However, an infinite matrix with periodic main diagonal can be a Jordan form only when it is diagonal.

3. As the elements of the main diagonal are pairwise different, it is clear that the Jordan form is the diagonal matrix with those elements in the main diagonal.

Now considering three cases we will prove Theorem 2. In the first case we have two possibilities - when the Jordan form is either a scalar matrix or a Jordan cell. We first note

Remark 2. If $R$ is a Riordan array and its Jordan canonical form $J_{R}$ is a scalar matrix, $J_{R}=c I$, then $R=c I$, since for any (invertible) $X$ we have $X^{-1} \cdot C I \cdot X=c I$.

While further considerations we are going to use the fact that if $R$ is a Riordan array and $J_{R}$ is its Jordan form, then there is a transition matrix $X$ so that

$$
R X=X J_{R}
$$

To finish case 1 . from Remark 1 we need one more observation.

Lemma 1. Suppose that $y, h \in \mathcal{F}_{1}$. If $y(h(t))=y(t)$, then $h(t)=t$.

Proof. The equation $y \circ h=y$ in the group $\mathcal{F}_{1}$ under composition has exactly one solution, i.e. $h=\mathrm{id}$.

Lemma 2. If $R$ is a Riordan array whose Jordan canonical form is a Jordan cell $J_{R}=(\alpha+t, t)$, then $R=(d(t), t)$ for some $d \in \mathcal{F}_{0}$.

Proof. Let $R=(d(t), h(t)), X=(x(t), y(t))$. Using (3) one obtains the system

$$
\left\{\begin{array}{l}
d(t) \cdot x(h(t))=x(t)(\alpha+y(t)) \\
y(h(t))=y(t)
\end{array}\right.
$$


From the second equation and Lemma 1 it follows that $h(t)=t$. Moreover, $x(t)$ can be, in fact, any series from $\mathcal{F}_{0}$, whereas $y(t)=-\alpha+d(t)$. Since $R$ and $J_{R}$ are similar, we must have $d_{0}=\alpha$. Thus, $y(t)$ is a well-defined series from $\mathcal{F}_{1}$.

One can check that Riordan matrices of the form $(d(t), t)$ have constant diagonals:

$$
r_{n k}=\left[t^{n}\right]\left(d(t) \cdot t^{k}\right)=\left[t^{n+1}\right]\left(d(t) \cdot t^{k+1}\right)=r_{n+1, k+1} .
$$

Now let's consider the case when the main diagonal forms a periodic sequence. This case is connected with a finite order. Finite order of formal power series was studied in [2], whereas finite order of Riordan arrays was thorougly investigated in [1,3] (also earlier in [4] but only the case of order 2). From [3] (Thm. 2) we get

Lemma 3. Suppose that $g(x)=g_{0}+g_{1} x+g_{2} x^{2}+\cdots$ and $F(x)=\omega x+F_{2} x+\cdots$ If $(g(x), F(x))$ has finite order in the Riordan group then $(g(x), F(x))$ is conjugate in $\mathcal{R}$ to $\left(g_{0}, \omega x\right)$.

Now, it is easy to conclude

Corollary 1. A Jordan form of a Riordan array $R=(d(t), h(t))$ is a diagonal periodic matrix if and only if $R=\alpha \tilde{R}$ where $\tilde{R}$ has a finite order.

Let's now discuss the last case from Remark 1.

Lemma 4. A Jordan form of a Riordan array $R$ is a diagonal non-periodic matrix if and only if the main diagonal of $R$ is a non-periodic sequence.

Proof. Let $J_{R}=(\alpha, \beta t)$. Then form (3) we get the system

$$
\left\{\begin{array}{l}
d(t) \cdot x(h(t))=\alpha \cdot x(t) \\
y(h(t))=\beta \cdot y(t) .
\end{array}\right.
$$

We will show that it is consistent. Expanding the first equation of (4)

$$
\left\{\begin{array}{l}
\alpha x_{0}=d_{0} x_{0} \\
\alpha x_{1}=d_{0} x_{1}+d_{1} x_{0} \\
\alpha x_{2}=d_{0} x_{2} \beta^{2}+d_{1} x_{1} \beta+d_{2} x_{0} \\
\cdots \quad \cdots \\
\alpha x_{n}=d_{0} x_{n} \beta^{n}+d_{1} x_{n-1} \beta^{n-1}+\cdots+d_{n-1} x_{1} \beta+d_{n} x_{0} \\
\cdots \quad \cdots
\end{array}\right.
$$

From the first equation of (5) and assumption that the transition matrix $X$ is a proper Riordan array (i.e. $x_{0} \neq 0$ and $y_{1} \neq 0$ ) one concludes that $d_{0}=\alpha$. Then from the $n$th equation of (5) it follows that

$$
x_{n} \alpha\left(1-\beta^{n}\right)=d_{1} x_{n-1} \beta^{n-1}+\cdots+d_{n-1} x_{1} \beta+d_{n} x_{0} .
$$


The latter and the fact that $\beta^{n} \neq 1$ for any $n$ imply that (5) can be solved inductively. Thus $x(t)$ exists in $\mathcal{F}_{0}$.

Consider now the second equation of (4) - according to [8] it, indeed has a solution, so we can omit the calculations.

Now one can see that Theorem 2 follows from Remark 2 Lemmas 2, 4 and Corollary 1.

\section{Examples}

Let us finish with some examples. First we present some Riordan arrays whose Jordan forms exist and transition matrices are Riordan arrays.

Example 1. The Riordan array

$$
R=\left(\frac{2+2 t}{\sqrt{1-4 t-4 t^{2}}+1}, \frac{\sqrt{1-4 t-4 t^{2}}-1}{2}\right)=\left[\begin{array}{cccc}
1 & & & \\
2 & -1 & & \\
4 & -4 & 1 & \\
12 & -12 & 6 & -1 \\
\vdots & & & \ddots
\end{array}\right]
$$

is an example for the first case of Theorem 2, i.e. is of degree 2 and we have

$$
X^{-1} R X=J_{R}=\tilde{I}=(1,-t)=\left[\begin{array}{ccccc}
1 & & & & \\
& -1 & & & \\
& 1 & & \\
& & -1 & \\
& & & \ddots
\end{array}\right]
$$

for

$$
X=\left(1+t, t+t^{2}\right)=\left[\begin{array}{lllll}
1 & & & & \\
1 & 1 & & \\
0 & 2 & 1 & \\
0 & 1 & 3 & 1 & \\
\vdots & & & & \ddots
\end{array}\right]
$$

Example 2. The Riordan array

$$
R=\left(\frac{1-t+t^{2}}{1-2 t+4 t^{2}}, 2 t\right)=\left[\begin{array}{ccccc}
1 & & & \\
1 & 2 & & \\
-3 & 2 & 4 & \\
-10 & -6 & 4 & 8 & \\
\vdots & & & \ddots
\end{array}\right]
$$

represents the second case of Theorem 2 - since $\beta$ is not a root of unity all its entries lying in the main diagonals are distinct. For this $R$ we have 


$$
X^{-1} R X=J_{R}=(1,2 t)=\left[\begin{array}{lllll}
1 & & & \\
& 2 & & \\
& 4 & & \\
& & 8 & \\
& & & \ddots
\end{array}\right]
$$

with

$$
X=\left(1-t+t^{2},-t\right)=\left[\begin{array}{ccccc}
1 & & & & \\
-1 & -1 & & & \\
1 & 1 & 1 & & \\
0 & -1 & -1 & -1 & \\
\vdots & & & & \ddots
\end{array}\right]
$$

Example 3. The array

$$
R=\left(3+3 t+3 t^{2}+t^{3}, t\right)=\left[\begin{array}{lllll}
3 & & & \\
3 & 3 & & \\
3 & 3 & 3 & \\
1 & 3 & 3 & 3 & \\
\vdots & & & \ddots
\end{array}\right]
$$

is a matrix from the third case of Theorem 2 -its diagonals are constant and its first subdiagonal is not equal to zero. In this particular case

$$
X^{-1} R X=J_{R}=(3+t, t)=\left[\begin{array}{lllll}
3 & & & & \\
1 & 3 & & & \\
1 & 3 & & \\
& 1 & 3 & \\
& & \ddots & \ddots
\end{array}\right]
$$

where

$$
X=\left(1-t^{2}, 3 t+3 t^{2}+t^{3}\right)=\left[\begin{array}{cccccc}
1 & & & & \\
0 & 3 & & & \\
-1 & 3 & 9 & \\
0 & -2 & 18 & 27 & \\
\vdots & & & & \ddots
\end{array}\right]
$$

From our results it follows that the problem with finding either the Jordan form or the transition matrix for some Riordan array $R=(d(t), h(t))$ in $\mathcal{R}$ appears when the main diagonal of $R$ is constant and either $h \neq$ id or $h=$ id but $d_{1}=0$. Let us have a look on those cases.

Example 4. First consider the case when the main diagonal is constant and $h \neq$ id. Let 


$$
R=\left(1+t, t-2 t^{2}\right)=\left[\begin{array}{ccccc}
1 & & & & \\
1 & 1 & & & \\
0 & -1 & 1 & & \\
0 & -2 & -3 & 1 & \\
\vdots & & & \ddots
\end{array}\right] .
$$

From Lemma 2.1 [9] we can conclude that this $R$ is similar to an array with the same main diagonal and the same first subdiagonal as $R$ and other entries equal to $0-\tilde{R}$. It can be also checked (we let ourselves leave it to the reader) that then one can find a diagonal array $D$ such that $D^{-1} \tilde{R} D=J_{R}=(1+t, t)$. Thus, $R$ considered in the group of lower triangular matrices has its Jordan form and the transition matrix is a lower triangular matrix $Y$. Let us check if $Y \in \mathcal{R}$, i.e. suppose $Y=(d(t), h(t))$. Then

$$
R Y=Y J_{R} \Leftrightarrow\left(1+t, t-2 t^{2}\right) *(d(t), h(t))=(d(t), h(t)) *(1+t, t) .
$$

The latter is equivalent to

$$
\left\{\begin{array}{l}
(1+t) d\left(t-2 t^{2}\right)=d(t)(1+h(t)) \\
h\left(t-2 t^{2}\right)=h(t) .
\end{array}\right.
$$

From Lemma 1 we already know that the second equation forces $h(t)=t$. Substituting it to the first one we get $(1+t) d\left(t-2 t^{2}\right)=d(t)(1+t)$ that, again by Lemma 1 , is a contradiction. Thus, there exists a lower triangular $Y$ so that $Y^{-1} R Y$ is $(1+t, t)$ but $Y \notin \mathcal{R}$.

Example 5. Let now $h(t)=t$, but $d_{1}=0$. We take a very simple example

$$
R=\left(1+t^{2}, t\right)=\left[\begin{array}{llllll}
1 & & & & \\
0 & 1 & & & \\
1 & 0 & 1 & & \\
0 & 1 & 0 & 1 & \\
\vdots & & & & \ddots
\end{array}\right]
$$

It can be noticed that for any $X=(d(t), h(t))$ the matrix $X^{-1} R X$ takes the form

$$
\left(1+(\bar{h}(t))^{2}, t\right)=\left(1+\bar{h}_{1}^{2} t^{2}+\cdots, t\right),
$$

i.e. either the first superdiagonal of $X^{-1} R X$ is zero although the second one is not. Obviously such an array is not a Jordan form.

Open Access. This article is licensed under a Creative Commons Attribution 4.0 International License, which permits use, sharing, adaptation, distribution and reproduction in any medium or format, as long as you give appropriate credit to the original author(s) and the source, provide a link to the Creative Commons licence, and indicate if changes were made. The images or other third party material in this article are included in the article's Creative Commons licence, unless indicated 
otherwise in a credit line to the material. If material is not included in the article's Creative Commons licence and your intended use is not permitted by statutory regulation or exceeds the permitted use, you will need to obtain permission directly from the copyright holder. To view a copy of this licence, visit http://creativecommons. org/licenses/by/4.0/.

\section{References}

[1] Cheon, G.-S., Kim, H.: The elements of finite order in the Riordan group over the complex field. Linear Algebra Appl. 439, 4032-4046 (2013)

[2] Cohen, M.M.: Elements of finite order in the group of formal power series under composition. arXiv:1804.00059v2

[3] Cohen, M.M.: Elements of finite order in the Riordan group. arXiv:1806.06432v2

[4] Kida, M.: On the involutions of the Riordan group. Funct. Approx. Commet. Math. 54(1), 19-23 (2016)

[5] Kostič, A., Petrovič, Z.Z., Pucanovič, Z.S., Roslavcev, M.: On a generalized Jordan form of an infinite upper triangular matrix. Linear Multilinear Algebra. https://doi.org/10.1080/03081087.2019.1632783

[6] Merlini, D., Nocentini, M.: Functions and Jordan canonical forms of Riordan matrices. Linear Algebra Appl. 565, 177-207 (2019)

[7] Shapiro, L.W., Getu, S., Woan, W.-J., Woodson, L.: The Riordan group. Discrete Appl. Math. 34, 229-239 (1991)

[8] Scheinberg, S.: Power series in one variable. J. Math. Anal. Appl. 31, 321-333 (1970)

[9] Słowik, R.: Expressing infinite matrices as products of involutions. Linear Algebra Appl. 438, 399-404 (2013)

Roksana Słowik

Faculty of Applied Mathematics

Silesian University of Technology

Kaszubska 23

44-100 Gliwice

Poland

e-mail: roksana.slowik@gmail.com

Received: September 23, 2020.

Accepted: April 8, 2021.

Publisher's Note Springer Nature remains neutral with regard to jurisdictional claims in published maps and institutional affiliations. 